\title{
Differences of statistical parameters of participants of water polo tournaments in Sisak and Sarajevo
}

\author{
Kenan Ademovic ${ }^{1}, \odot$ Edin Mirvic $^{1}, \odot$ Dzan Lemes $^{1}, \odot$ Elvira Niksic $^{2}$
}

${ }^{1}$ Faculty of Sports and Physical Education, University of Sarajevo, Bosnia and Herzegovina. ${ }^{2}$ Faculty of Educational Sciences, University of Sarajevo, Bosnia and Herzegovina.

\begin{abstract}
This paper aims to analyze the statistical parameters and to determine the differences between the water polo tournament in Sisak and the water polo tournament in Sarajevo. Situational efficiency utilizing certain variables shows us the most important parts of the game during a water polo match. Statistics is a scientific discipline that studies the methods of collecting, collating, analyzing, and interpreting data. The sample consists of 336 water polo players, from six clubs of two age categories from the league of Canton Sarajevo and six clubs of two age categories from the Sisak International Tournament, in the "junior and cadet" categories for men born in 2003 and 2004, 2005. The sample of variables consists of 9 tests to assess situational efficiency in a water polo game that defines the main characteristics of a water polo match. The following will explain the observed variables: goals scored, attempt to score, goal from action, center to score, anchor to score, penalty, attempt from penalty defended by the goalkeeper or player missed, foul on the counter-attack, sprint - counter with a goal scored. The results of the $\mathrm{H}$ square test show that there are statistically significant differences ( $p$.000) in the analyzed segments of the water polo match. It can be concluded that the teams in the tournament in Sisak are better organized because in every aspect they have achieved better results in comparison to the Sarajevo tournament. The teams from Sisak were better and faster swimming because a lot of goals were scored by counterattack, more attempts to shoot at goal (Sisak), fewer penalties, which means that in Sarajevo is rough play and greater success of winning teams in penalties.
\end{abstract}

Keywords. Competition, differences, notation analysis, technique.

\section{Introduction}

New technologies in sports and the development of the natural sciences influence the improvement of sport results and the improvement of the training process. Water polo is a water sport played with ball for the male and female population, which today enjoys great popularity around the world and is the oldest team discipline of the Olympic Games with football. Water polo is a team sport that belongs to a group of polystructural complex kinesiological activities and is different from other team sports, because it is played in a completely different and specific environment - water (Mirvić \& Nurković, 2018). Water polo player during the match, within the rules of a certain playground (30$22 \mathrm{~m}$ ) with two goals, moving in different directions, different speeds, and different distances. Different movement techniques are used in the game, with and without the ball. They often stop and move again. Development and improvement of a modern water polo game, accelerated rhythm, and pace, situational activities and response on specific occasions, new requirements regarding the anthropological dimensions of water polo players and a continuous flow of actions at all stages of the game, require serious approach and work on improving water polo technical and tactical knowledge for all selection. In their research, Platanou \& Geladas (2006) concluded that shortterm training in terms of playing tactics, before significant competitions, did not show sufficient efficiency, while long-term preparations of the 
players, regardless of the position they played, contributed greatly to the team victory. Studies that have identified the complexity of water polo games for men (Polglaze et al., 2008) indicate a high intensity of repetitive technical elements of players, due to the specificity in which the activities (aquatic environment) and combat with rival players take place (Mirvic et al., 2014). In this paper, the authors analyzed the difference between winning and losing teams in top water polo at the Men's World Championship. Chi-square test results showed that there were statistically significant differences $(p$ $.000)$ in the analyzed segments of water polo matches. The teams that prevailed were more adept at motor skills and had faster swimmers, due to the large number of goals scored from the counterattack. The authors also concluded that there were more fouls (rough play) in the game compared to earlier water polo matches. If a serious offense is committed within a 5-meter line on the striker, the offender gets a personal foul and the opposing team starts to take a penalty throw. It is kicked at the opponent's goal from a 5-meter line and can be played by any player except the goalkeeper. Judges simultaneously mark a hit by raising the white and red flags (Kermeci, 2017). From all the above, this paper aims to analyze the statistical parameters and to determine the differences between the water polo tournament in Sisak and the water polo tournament in Sarajevo. Situational efficiency utilizing certain variables shows us the most important parts of the game during a water polo match. Statistics is a scientific discipline that studies the methods of collecting, collating, analyzing, and interpreting data.

\section{Methods}

\section{Subjects}

The sample consists of 336 water polo players, from six clubs of two age categories from the league of Canton Sarajevo and six clubs of two age categories from the Sisak International Tournament, in the "juniors and cadets" categories for men of 2003 and 2004, 2005. The survey included only those respondents who participated in these two competitions, with the appropriate conditions required for testing in this research.

\section{Procedures}

The sample of variables consists of 9 tests to assess situational efficiency in a water polo game that defines the main characteristics of a water polo match. The following will explain the observed variables:

Goals scored: This variable indicates the total number of goals scored during a tournament match. The way to collect this variable was to observe the official scoreboard at the meetings, and it indicated when the ball crossed the goal line from the correct shot and the referee conceded the goal.

Attempts on goal: Variable attempts on goal indicate any intent to shoot at a goal from a fair play where the player was prevented by a goalkeeper, goal frame, or defensive player who interfered when trying to score.

Goal from action: Indicates the correct passage of the ball across the goal line in the drafted attack. This meant that only goals were taken into account in these variables, where the team had a clearly defined leverage in the attack during which the goal was scored.

Center goal: Center goal indicates a player who is in the center back position between the left and right back and the players in the center back position are predominantly taller and strong shot. The variable indicates the goal scored by a player in the center position. The goals scored by the players of the center who were the creators of the game played by a certain team were taken correctly.

Anchor scored a goal: Indicates an anchor player. This player is closest to the goalkeeper of the opposing 
team and faces back to him. His role is very demanding because he constantly has one of the players on his back. The variable indicates the correct goal scored by the player from the anchor position. Also, as with the center back, goals scored in the attack were taken into account.

Penalty: This variable indicates the penalty awarded by the referee. The penalty line is 5 meters from the goal line. At the referee's sign, the player shoots at goal. The total number of penalties taken in both tournaments was taken into account.

Attempt from a penalty shot which was denied by goalkeeper or the player missed: Indicates a variable similar to the previous one, except that the total number was taken from the selection of missed penalties where the player performing the penalty was prevented by the goalkeeper or the player missed.

Foul in counter-attack: Denotes the interruption of a game in which a player stopped another player by incorrectly stopping, ie. the referee flagged a foul play by a player who stopped the player intended to counter-attack. All offenses awarded were counted as indicating a counterattack by a particular team.

Sprint: Counter with the goal scored - indicates a variable in which certain player sprints into space with speed and scores when taking a ball from the opposing team. Only those goals were taken into account where the ball was seized behind half of the field and by the addition or guidance of the ball to the goal of a particular player, the goal was scored. Approvals from appropriate institutions and individuals were used to ensure the regularity of this research process. Based on the variables already identified, data collection could be approached to assess situational efficiency in water polo players. The results were then processed by statistical methods, which made the difference between the two tournaments.

\section{Research Description}

In order to measure the differences between the water polo tournament in Sisak and the water polo tournament in Sarajevo, we had to get the consent of the ethics committee and that it was done according to the Helsinki Declaration. All subjects were tested under the same conditions (air temperature was from 26 to $30^{\circ}$, water temperature was from 24 to 26 degrees Celsius in Sarajevo and Sisak). The research was realized so that all matches were recorded with a video camera (tournament in Sisak and tournament in Sarajevo with the written consent of the parents of children and clubs). After collecting data from the tournaments in Sisak and Sarajevo, we analyze each match (separately tournament in Sisak, and separetly the tournament in Sarajevo), then the results are entered into the measurement lists, and the data are processed by appropriate methods for this research work.

\section{Data Processing Methods}

Data processing methods were selected following the characteristics and size of the sample of respondents, the hypotheses in explaining this research, as well as the subjects and problems, and the purpose of the research. Microsoft Office Excel 2007 software was used for data entry. The statistical package IBM SPSS Statistics (version 20.0, SPSS Inc, USA) was used for statistical data processing. First, descriptive statistical methods were used, and intercorrelation and finally to determine differences between frequencies from the Sisak and Sarajevo Canton tournaments, Chi-Square test at the statistical significance level of $5 \%$ was used. The results are presented in tables and graphs.

\section{Results}

Table 1 shows the results in the differences between the frequencies of the variables applied at the 2017 tournament in Sisak and Sarajevo Canton. The 
results of the chi square test clearly show that there are statistically significant differences of 0.024 in the analyzed segments of the water polo match. Analyzing the individual variables, it can be noticed that a total of $587(15.85 \%)$ goals were scored, of which $381(17.83 \%)$ in Sisak and $206(13.48 \%)$ in Sarajevo Canton. Teams from Sarajevo Canton tried to score $430(27.52 \%)$, and teams from Sisak tried $739(34.99 \%)$, a total of $1169(31.76 \%)$ attempts to score. Teams from Sisak scored goals from the action $196(9.02 \%)$ times, and teams from Sarajevo Canton $75(4.87 \%)$ times, a total of 271 (7.32\%).
Central players in the Sarajevo Canton tournament scored 15 goals $(0.96 \%)$, and Central players in the Sisak tournament scored 90 (4.24\%), a total of 105 (2.84\%). Anchors from the tournament in Sisak scored 105 (4.83\%) goals, Anchors from the tournament in Canton Sarajevo scored 46 (2.94\%) total $151(4.08 \%)$ goals. Penalties were scored in total by $92(2.48 \%)$, of which teams from the tournament in Sisak scored $60(2.82 \%)$, and teams in Sarajevo Canton scored $32(2.04 \%)$ goals from penalties.

Table 1

Results from the tournament in Sisak and Canton Sarajevo.

\begin{tabular}{lccc}
\hline Variables & Sisak Tournament & $\begin{array}{c}\text { Canton Sarajevo } \\
\text { Tournament }\end{array}$ & Total \\
\hline Goals scored & 381 & 206 & 587 \\
Attempts on goal & $17.83 \%$ & $13.48 \%$ & $15.85 \%$ \\
& 739 & 430 & 1169 \\
Goal from action & $34.99 \%$ & $27.52 \%$ & $31.76 \%$ \\
& 196 & 75 & 271 \\
Center goal & $9.02 \%$ & $4.87 \%$ & 105 \\
& 90 & 15 & $2.84 \%$ \\
Anchor scored a goal & $4.24 \%$ & $0.96 \%$ & 151 \\
Penalty & 105 & 46 & $4.08 \%$ \\
Attempt from a penalty shot which was & $4.83 \%$ & $2.94 \%$ & 92 \\
denied by goalkeeper or the player missed & 60 & 32 & $2.48 \%$ \\
Foul in counter-attack & $2.82 \%$ & $2.04 \%$ & 176 \\
Sprint - counter with the goal scored & 78 & 98 & $4.75 \%$ \\
& $3.59 \%$ & $6.27 \%$ & 914
\end{tabular}

Chi-square $=230 ;$ Degrees of freedom $=9 ;$ Probability $=0.024$ 
Attempts from a penalty shot which was denied by the goalkeeper or the player missed a total of 176 $(4.75 \%)$ defenses or teams from the Sarajevo Canton did not score $98(6.27 \%)$ and teams from Sisak 78 $(3.59 \%)$. Foul in the counterattack was made by teams from Sisak $366(16.94 \%)$, and teams from Sarajevo Canton 548 (35.57\%) in total 914 (24.88\%). Sprint with counterattack and goal scored was a total of $224(6.05 \%)$, of which teams from Sisak had $125(5.75 \%)$ and teams from Sarajevo Canton 99 $(6.34 \%)$.

\section{Discussion}

Chi square test used when quantitative or distribution data deviate significantly from the normal distribution. The value of the Chi - square test in our case is 0.024 . Considering the variables used in the paper, we have found that there are significant differences between the water polo tournament held in Sisak and the water polo tournament held in Sarajevo, which confirms our hypothesis that there is a statistically significant difference between the statistical parameters of the Sisak water polo tournament participants and the water polo tournament participants is being held in Sarajevo. As noted in the paper, a total of 52 matches were observed, which is 26 matches per tournament. The biggest difference was observed in the variables: "try to score", "goals scored" and "goal from action", which indicates that on average, the teams in the Sisak tournament scored 14.65 goals, which is significantly more in compared to the tournament held in Sarajevo where the average number of goals was 7.92. The faster game was realized at the tournament in Sisak because the difference between the numbers of attempts to score more than the tournament was held in Sarajevo by 309 attempts. Then, the goals scored are justified by 175 more goals compared to the tournament held in Sarajevo. Mirvic et al. (2014) in their work also analyzed the difference between winning and losing teams in top water polo at the Men's World Championship. The teams that prevailed were more adept at motor skills and had faster swimmers, and the dynamics were on the side of the winning team that won the finals and showed better results. Lupo et al. (2013) in their study aimed to compare technical and tactical aspects between the winning and losing teams, differences also emerged for the occurrence of the action (even the counterattack). Mirvic et al. (2019) concluded in their research that only unbalanced games are affected by technical and tactical aspects that can discriminate between winning and losing results at women's water polo Olympic Games. One of the most important variables is the goal-from-action variable, which proves to us that the organization of the game is being carried out on a pre-defined tactic that the water polo players from the Sisak tournament realized 121 goals more compared to the tournament held in Sarajevo. This tells us that at the tournament in Sisak the ball was passed more than halfway through the field and the attack with a defined fan was set up within the first $15-20$ seconds, thus the water polo players from the tournament held in Sisak had more time to realize the attack. Then the goal scored from the action shows us that the water polo players at the Sisak tournament are technically and tactically more prepared than the water polo players from the tournament held in Sarajevo. Looking at the water polo players from the Sisak tournament who showed organized play and based the game on actions with the whole team compared to the water polo players from Sarajevo who based the game on the individual abilities of certain water polo players and thus based the game on individual players. The following observed variables with significant differences are: "center goal", "anchor goal" and "penalty". As evidenced in their research (Mirvić et al., 2011), the winning teams had counter-attacking tactics because they had better swimmers and better swimming, so the defense team committed offenses that made the winning team better and the game was based on the central player because the central player is the organizer of the game. As already shown in the results, the players at the center 
position in the tournament held in Sisak scored 75 goals more than the players at the center position from the tournament held in Sarajevo Canton. Anchor players at the tournament held in Sisak scored 59 more goals than players at the anchor position in the tournament held in Sarajevo. The reason for this comes from the fact that the game in the tournament in Sisak was based on tactical attacks so that more players from the positions of center and anchor were compared to the players from the tournament held in Sarajevo who occasionally had defined attacks, and therefore few hits from these positions which again tells us that water polo players from the tournament in Sisak have better motor skills primarily speed - strength. As it is already known that the players in the anchor position are the most responsible for the construction of the attack and the players with the biggest load they carry in the game, it can be noticed that there are no adequate players in the water polo tournament in Sarajevo for such a demanding position, so the game was based more on the counterattack and a large number of hits were thus realized. One of the significant variables is the "counter-attack foul" which shows us that the number of fouls at the tournament held in Sarajevo was 182 fewer than the tournament held in Sisak. The reason for this is the speed endurance of some water polo players from the tournament held in Sarajevo Canton because the opposing players were unable to cut off the counterattack against the players from the tournament held in Sisak, where the players were stopped in time by the opposing team in an attempt to counter-attack. ArgudoIturriaga et al. (2009) in a study analyzing the results that there are significant statistical differences in the thirty-six value parameters that are attack and defense in the female categories and forty-six men between the winning and losing teams. If we compare the results of this study with those of Argudo-Iturriaga (2000), Argudo-Iturriaga \& Lloret (1998), and Argudo-Iturriaga \& Lloret (1999), where the subject of analysis is focused on tactical assessment in water polo at the 1997
European Championships, it is clear that between winners and losers, in women's water polo, are at the level of statistical significance until, 05. This tells us that teams from the Sisak Tournament are collectively prepared and experienced enough to know on their counter-attack intentions on time. In the following observed variables, there is also some difference between the parameters in the two tournaments. We can see that the water polo players from the Sisak tournament were better in penalties and thus had 20 fewer misses than the water polo players from the Sarajevo tournament. The next observed parameter "sprint - counter with the goal scored" tells us about the physical preparedness of water polo players, so water polo players from Sisak showed significantly better speed abilities. As a result, the Sisak water polo players were more successful in 26 sprints - goals scored. By looking at all the variables, we can conclude the difference between the water polo players in these two tournaments. Water polo players from Sisak have shown a varied and interesting game that can serve the coaching staff of clubs from the tournament in Sarajevo Canton. This is justified by statistical methods that show clear differences between the parameters observed in the present study. Intercollation shows us the interconnectedness of the observed variables, that is, each variable has a significant effect on the other observed variables. The author believes that a significant difference would be made by reducing the size of the pitch at the Sarajevo tournament. This would make the game much more interesting as it would be easier to organize attacks in the fan area, thus increasing the number of shots on goal and making players from the anchor and center positions more in the focus of attack. Also, all these results are good indications that more attention is paid to the Sarajevo Canton when planning and programming the training process. In their study, Argudo-Iturriaga et al. (2007) at the 10th World Championships in the male population between losers and winners proved the difference in determination to action and precision of addition and shot. It can be concluded that a 
significant difference would be made by reducing the size of the pitch in the Sarajevo tournament. This would make the game much more interesting as it would be easier to organize attacks in the fan area, thus increasing the number of shots on goal and making players from the anchor and center positions more in the focus of attack. Also, all these results are good indications that more attention is needed to pay to the Sarajevo Canton when planning and programming the training process.

\section{Conclusion}

The results of this research show that there is a statistically significant difference between the statistical parameters of participants in the water polo tournament in Sisak and participants of the water polo tournament held in Sarajevo. Sarajevo Canton and 6 clubs from the Sisak International Water Polo Tournament in the categories of "juniors and cadets" for men of 2003 and 2004, 2005. It can be concluded that the teams in the Sisak tournament are better organized because they achieved better results in every aspect in contrast to the Sarajevo tournament. The teams from Sisak were better and faster in swimming because a lot of goals were scored by counter-attack, more attempts to shoot at goal (Sisak), fewer penalties, which means that in Sarajevo rough play and better success of winning teams in penalties. As expected, the dynamics of the game was better in the tournament in Sisak, as well as competition on the field, which was shown by the results. From all of the above, it can be said that in Sarajevo - Bosnia and Herzegovina there has been a positive development in terms of the technical part, but the organization of tactics is still lagging behind. The results can be used in water polo clubs, the Faculty of Sport and Physical Education, and similar institutions dealing with this issue. The results of the survey may provide a good basis for further research projects aimed at improving water polo in Sarajevo - Bosnia and Herzegovina.

\section{Acknowledgment}

The authors would like to thank every participant for his effort and time.

\section{Funding}

No external funding was received for this study.

\section{Declaration of Interest}

The authors report no conflict of interest.

\section{References}

Argudo-Iturriaga, F.M., Roque, J.I.A., Marín, P.G., \& Lara, E.R. (2007) Influence of the efficacy values in counterattack and defensive adjustment on the condition of winner and loser in male and female water polo. International Journal of Performance Analysis in Sport, 7(2), 81-91.

Argudo-Iturriaga, F.M. (2000). Model evaluation tactics in opposition to sports cooperation. Study práxico Waterpolo him. Doctoral Thesis, University of Valencia, Spain.

Argudo-Iturriaga, F.M., \& Lloret M. (1998). Coefficients of offensive and defensive performance in equal numerical feminine Waterpolo-97 in Seville. Water Management, 42, 16-30.

Argudo-Iturriaga, F.M., \& Lloret, M. (1999). Coefficients of offensive and defensive performance in Waterpolo feminine. Technical Communication, 2, 45-67.

Argudo-Iturriaga, F.M., Ruiz, E., \& Alonso, J.I. (2009). Were differences in tactical efficacy between the winners and losers teams and the final classification in the 2003 water polo world championship? Journal of Human Sport and Exercise, 4, 142-153.

Kermeci, R. (2017). Pravila vaterpola. Sportilus.

Lupo, C., Condelo, G., Capranica, L., \& Tessitore, A. (2013). Women's water polo World Championships: Technical and tactical aspects of winning and losing teams in close and unbalanced games. J Strength Cond Res, 28(1), 210-222.

Mirvić, E., Kazazović, B., \& Aleksandrović, M. (2011). Differences between winning and losing teams from 
world water polo championship for women. Scientific Journal of Sport and Physical Education, 13(2), 38-42.

Mirvić, E., \& Nurković, N. (2018). Vaterpolo. Fakultet sporta i tjelesnog odgoja, Sarajevo.

Mirvić, E., Rašidagić, F., Nurković, N., Kajmović, H., \& Lupo, C. (2019). Only unbalanced games are affected by technical and tactical aspects able to discriminate winning and losing performance in women's water polo Olympic Games. Sport Sciences for Health, 1-8.

Mirvić, E., Rašidagić, F., \& Osmo, B. (2014). Differences between winners and defeated teams in water polo at the world championship for men. Sport Science, 7(1), 108-115.

Platanou, T., \& Geladas, N. (2006). The influence of game duration and playing position on intensity of exercise during match-play in elite water polo players. Journal of Sports Sciences, 24(11), 1173-1181.

Polglaze, T., Rechichi, C., Tan, F., Hankin, S., \& McFadden, G. (2008). The repeat high-intensity activity characteristics of elite women's water polo [Abstract]. The Verona-Ghirada Team Sport Conference, Treviso, Italy. 